\title{
Intermédialités
}

Histoire et théorie des arts, des lettres et des techniques

Intermediality

History and Theory of the Arts, Literature and Technologies

\section{L'amour, en ruines : notes sur quelques photogrammes du film de Bill Morrison Light is Calling}

\section{André Habib}

Numéro 4, automne 2004

Aimer

Loving

URI : https://id.erudit.org/iderudit/1005481ar

DOI : https://doi.org/10.7202/1005481ar

Aller au sommaire du numéro

Éditeur(s)

Centre de recherche sur l'intermédialité

ISSN

1705-8546 (imprimé)

1920-3136 (numérique)

Découvrir la revue

Citer ce document

Habib, A. (2004). L'amour, en ruines : notes sur quelques photogrammes du

film de Bill Morrison Light is Calling. Intermédialités / Intermediality, (4),

157-162. https://doi.org/10.7202/1005481ar d'utilisation que vous pouvez consulter en ligne. 


\title{
L'amour, en ruines: notes sur quelques photogrammes du film de Bill Morrison Light is Calling
}

\author{
ANDRÉ HABIB
}

En 1945, André Bazin écrivait:

[L]e cinéma apparaît comme l'achèvement dans le temps de l'objectivité photographique. Le film ne se contente plus de nous conserver l'objet enrobé dans son instant comme, dans l'ambre, le corps intact des insectes d'une ère révolue, il délivre l'art baroque de sa catalepsie convulsive. Pour la première fois, l'image des choses est aussi celle de leur durée, et comme la momie du changement ${ }^{1}$.

Près de soixante ans après qu'elle eut été énoncée, cette formule célèbre se trouve tout à la fois confirmée et contredite par le «destin des images» cinématographiques. Si, embaumé par l'appareil de captation, le temps réel des choses que la caméra enregistre se «soustrait à sa propre corruption", leur image se trouve tôt ou tard soumise à un autre type de décomposition : celle de la pellicule cinématographique sur laquelle se formule fatalement le passage des ans. Dans les voûtes des cinémathèques, dans les caves et greniers de collectionneurs, longtemps jugé obsolètes par les archivistes, les historiens et les « exhibiteurs », repose un stock inimaginable d'images pourrissantes: fragments anonymes, bobines abîmées, rouleaux rongés par la moisissure. Ces lambeaux de pellicules, voués à l'oubli, forment depuis une vingtaine d'années la matière première des pratiques de plusieurs artistes ${ }^{2}$ qui, tout en empruntant des voies

1. André Bazin, «Ontologie de l'image photographique» [1945], dans Qu'est-ce que le cinéma?, Paris, Les Éditions du Cerf, 1997 [1985], p. 14.

2. On notera parmi les plus remarquables les cinéastes Peter Delpeut (Lyrisch nitraat, 1990), Jürgen Reble (Passion, 1989-1990), Angela Ricci-Lucchi et Yervant Gianikian (Dal 
différentes, s'emploient à exhumer ces fragments et à leur donner une seconde vie, une nouvelle visibilité. Chacune de ces œuvres est d'ailleurs traversée par un instant décisif où, au hasard des recherches, comme par un coup de foudre, l'artiste se trouve soudainement happé par la beauté plastique ou la puissance d'évocation de ces images rescapées, brûlées par le temps. Le plaisir esthétique que suscitent ces images en ruines repose en effet sur la "valeur d'ancienneté » de la pellicule filmique (patine du temps, scories ombreuses, couleurs éclatantes), la mémoire historique qu'elles portent, et les refigurations surprenantes qu'engendre l'usure du support filmique. On pourrait dire que les films du newyorkais Bill Morrison explorent toutes les facettes de cette nouvelle « esthétique des ruines ».

Arrivé au cinéma par le détour de la peinture, c'est en plasticien tout d'abord qu'il envisage son travail cinématographique. Depuis le début des années 1990, il poursuit, très souvent avec la collaboration de la compagnie Ridge Theater de New York, et de compositeurs-musiciens (Michael Gordon, Bill Frisell), une œuvre singulière qui exploite, entre autres, le rythme et les textures visuelles de la pellicule détériorée, créant une fusion hypnotique d'images et de sons ${ }^{3}$. Morrison s'interroge sur le sens de l'histoire, de la mémoire des images et de leur oubli (The Film of Her, 1996; Decasia, 2002), ou encore sur l'impact proprement «attractif» du cinéma sur nos sens (Footprints, 1992; The Death Train, 1993). Tout particulièrement, ses «films d'archives» - ou found footage selon l'expression consacrée - , appellent une réflexion sur la «mémoire impossible de la pellicule ${ }^{4}$ qui, en se décomposant superbement, révèle néanmoins l'importance de sa préservation et du temps propre au cinéma.

En 2001, les employés de la Library of Congress à Washington présentent à Morrison une copie nitrate teintée, fortement abîmée, d'un film de James Young, The Bells (1926), qu'ils s'apprêtaient à détruire. En remontant ce matériau filmique, en ajoutant une bande sonore, et en rephotographiant à l'aide d'une tireuse optique chaque photogramme afin de ralentir le défilement des images et de mettre en valeur les effets plastiques engendrés par la décomposition

polo all'equatore, 1987, Su tutte le vette è pace, 1998), ainsi que le photographe-plasticien Éric Rondepierre (Précis de décomposition, Moires, Les trente étreintes, 1993-1999).

3. La compagnie de production de Bill Morrison porte d'ailleurs le nom de Hypnotic Pictures.

4. Propos rapportés par Élisabeth Lebovici, «Mes films pourriront aussi. Entretien avec Bill Morrison », Libération.fr, 11 juin 2003, <http ://www.liberation.fr/imprimer.php?/ Article $=116790>$ 
de l'émulsion (mais sans jamais retoucher les images à proprement parler), Morrison tira de cette copie originale deux films originaux: The Mesmerist (2003) et Light is Calling (2004). C'est de ce dernier film que sont extraits les vingt photogrammes qui composent le présent dossier.

Dans l'une des scènes du film de Young, un officier de cavalerie tombe sous le charme de la fille d'un aubergiste puis tente de - et finit par - la séduire. Cette scène, qui ne dure que quelques instants dans le film original, est métamorphosée dans Light is Calling, grâce à un procédé complexe ${ }^{5}$, en un riche poème visuel de huit minutes accompagné par une partition pour orchestre de Michael Gordon. Le temps ainsi dilaté, le découpage de l'action se résume à quelques traits minimaux: plans de la cavalerie, jeux de regards de la jeune fille et du jeune homme, rencontre du couple au centre de l'image, sortie du cadre par la droite. Cette description n'épuise pas toutefois ce qui se passe dans le film et qui a précisément à voir avec ce qui s'est passé sur la pellicule. Avec la décomposition du nitrate sont apparus de larges trous, crevasses, bouillons roses, blancs, pourpres, noirs, qui ont modifié le mouvement des corps et l'expression des visages, dégoulinant ou éclatant en girations spectaculaires, pris dans le flux mouvant des images. La pellicule ruinée, tout comme, à une autre époque, les pierres imagées ${ }^{6}$, crée des transformations visuelles qui se prêtent au jeu des analogies et des métaphores, accusant certains traits de l'image, approfondissant ou surdéterminant parfois les scènes représentées: pathétisme, caricature, parodie, mélancolie, affectation, mascarade, autant d'effets dramatiques que la décomposition de la pellicule engendre.

Si l'image animée décomposée libère des virtualités plastiques inattendues, il en va tout autant, sinon plus, de la présentation de photogrammes fixes. Elle appelle encore une autre appréciation de ces images et confirme l'intérêt que peut représenter le photogramme, que Roland Barthes a décrit en 1970 comme un «artefact majeur». À ce propos, Barthes en parlait comme de la

5. Chaque photogramme du film original a été multiplié quatre fois à la tireuse optique. Chaque série de quatre photogrammes a ensuite été surimposée sur les deux premiers photogrammes de la série suivante, et ainsi de suite. Ceci produit un étrange effet de fondu enchaîné continu, et contribue à cet effet de flottement fantomatique sur lequel repose l'efficace visuelle du film.

6. Sur ce point, voir Jurgis Baltrušaitis, Aberrations: essai sur la légende des formes, 1. Les perspectives dépravées, Paris, Éditions Flammarion, coll. «Idées et recherches», 1983 [1957], p. 54-88. 
trace d'une distribution supérieure des traits dont le film vécu, coulé, animé, ne serait en somme qu'un texte, parmi d'autres. [...] film et photogramme se retrouvent dans un rapport de palimpseste, sans qu'on puisse dire que l'un est le dessus de l'autre ou que l'un est extrait de l'autre?.

Ce qui est vrai du rapport de palimpseste entre le photogramme et le film l'est également pour la relation que nouent cette image et son support matériel, dans lequel les corps semblent à la fois se lover et agoniser. Le vieillissement de la pellicule produit de nouvelles possibilités de signification, de la même manière qu'un autre sens (ce que Barthes appelle «le troisième sens », ou le «sens obtus») apparaît à l'analyse du photogramme: ces photogrammes en ruines rendent visible un nouveau récit amoureux, par-dessus ou par-devant le premier, reproduisant et dramatisant les différents instants de la rencontre des amants, comme si chaque image en captait un moment: l'approche, l'attention, l'hésitation, la déchirure, le timide acquiescement, la cristallisation amoureuse, la fuite, l'abandon, l'embras(s)ement, etc. Les effets d'empâtement, de lavis, de coulures que le hasard a produits rompent la continuité des gestes et développent en autant de tableaux une nouvelle intrigue, tantôt féérique ou onirique, tantôt cauchemardesque ou horrifiante.

Tout comme dans la série du photographe français Éric Rondepierre, Les trente étreintes (1997-1999, fig. 1-2), les corps des amoureux sont plongés dans une agitation, une tourmente qui a de toute évidence peu à voir avec le mouvement initial de la scène. La force de ces deux séries de photogrammes est sans doute de donner à ces accidents matériels une autonomie esthétique. La matière et l'image sont à ce point confondus qu'il serait en effet difficile d'imaginer l'état primitif de ces photogrammes, ou encore de distinguer ce qui relève de l'image, et ce qui est le fruit de la décomposition du support. Ces images en mouvement suspendu se présentent comme des compressions de durée stratifiées, déployées en autant de figures de matière, ou de figurations matérielles. Tout comme un temple tombé en ruines ou une fresque délitée ${ }^{8}$, les accidents

7. Roland Barthes, «Le troisième sens. Notes de recherches sur quelques photogrammes de S.M. Eisenstein", Cahiers du cinéma, 1970, repris dans L'obvie et l'obtus, Essais critiques III, Paris, Éditions du Seuil, coll. «Tel quel», 1982, p. 60.

8. «Il faut ruiner un palais pour en faire un objet d'intérêt. » (Denis Diderot, Salons de 1767, repris dans Salons III : ruines et paysages, Else Marie Bukdhal, Michel Delon, Annette Lorenceau (éds.), Paris, Éditions Hermann, coll. «Savoir : Lettres», 1995 [1767], p. 348) 

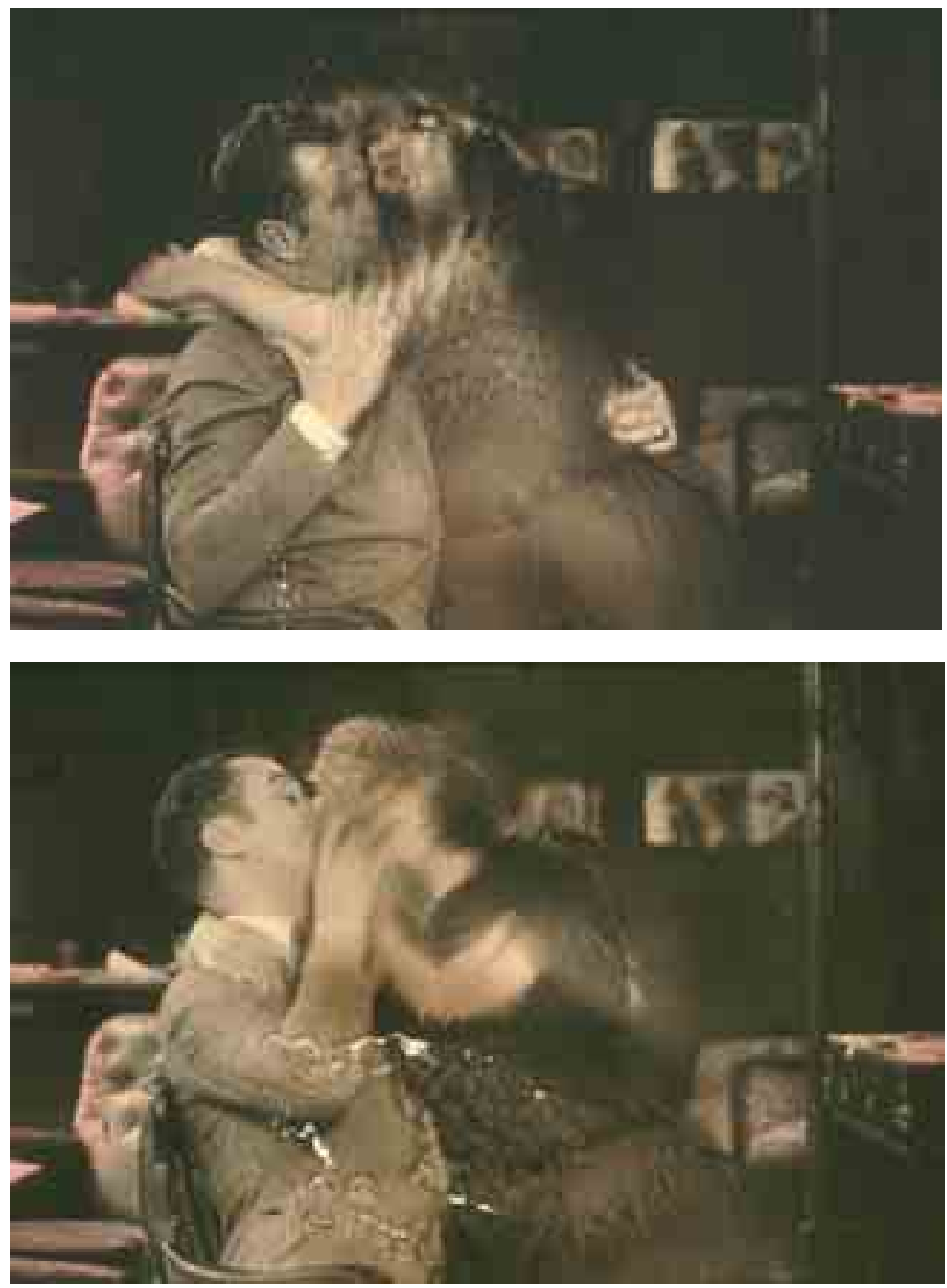

Fig. 1-2, Éric Rondepierre, Étreintes $n^{o s} 15$ et 22 (Les trente étreintes), 1997-1999, Cibachrome sur aluminium, $40 \times 60 \mathrm{~cm}$ (avec l'aimable permission de l'artiste). 
du temps ont réalisé une œuvre à part entière, que le cinéaste-chiffonnier rapièce, découd, donne à voir, et qui peut évoquer les têtes convulsées des polyptyques de Francis Bacon, une valse de fantômes ou l'iconographie des contes de fées.

Il serait possible d'étendre la remarque de Dominique Païni, formulée il y a quelques années à propos de la fièvre restauratrice et de la revalorisation du fragment filmique dans les cinémathèques, et affirmer qu' « un véritable “imaginaire des ruines"9 » s'est emparé du cinéma depuis une vingtaine d'années. Cet imaginaire est certainement lié à un amour des images, mais encore plus, il semble, à un amour - parfois même fétichiste ${ }^{10}$ — pour la pellicule cinématographique (son odeur, son toucher, sa lumière) que les supports numériques sont censés rendre bientôt obsolète. La pellicule se voit aujourd'hui conférés tous les attraits de l'aura, dont Benjamin l'avait autrefois privée, précisément parce qu'elle est toujours en train de disparaître, de s'étioler, de mourir ${ }^{11}$. Les films de Bill Morrison problématisent cette dialectique entre apparition et disparition en en faisant une spécificité du cinéma. Son œuvre nourrit et prolonge toutes les réflexions contemporaines sur la survivance du cinéma et l'amour des images. À l'évidence, cet amour se dit aussi en ruines.

9. "C'est ainsi qu'un véritable "imaginaire des ruines" a envahi les cinémathèques. » (Dominique Païni, «La résurgence du fragment», dans Le cinéma, un art moderne, Paris, Éditions des Cahiers du cinéma, 1997, p. 144)

10. Ce fétichisme est pleinement assumé, entre autre, par Paolo Cherchi Usai quand il écrit: «Is this fetishism? Yes indeed, insofar as fetishism - the art of establishing a physical relationship with the object of desire beyond the fulfilment of pleasure - can lead to a true form of knowledge. » (Paolo Cherchi Usai, «An Epiphany of Nitrate», dans Roger Smither (dir.), This Film is Dangerous, Bruxelles, Fédération internationale des archives du film, 2002, p. 129)

11. Le même champ sémantique a été depuis longtemps déployé pour parler du cinéma en tant qu'art. Sans doute un travail de fond reste à être mené sur les coïncidences de ces deux champs à travers l'histoire du cinéma qui analyserait les incidences entre ces discours sur "la mort du cinéma» et les ruptures médiatiques que le cinéma a traversées. 